\title{
La credibilidad periodística en jaque: conexión entre propaganda y fake news
}

\author{
Journalistic credibility at stake: connection between propaganda and fake \\ news
}

\author{
Dra. Magaly Parreira do Prado \\ Universidade de São Paulo | Cidade Universitária, São Paulo - SP| Brasil \\ |https://orcid.org/0000-0003-2792-0264| magalyprado@usp.br
}

Fechas | Recepción: 15/02/2021 | Aceptación: 30/05/2021

\section{Resumen}

El tema es la emergencia de las fake news tumultuando la cultura democrática y desacreditando el periodismo. El objetivo es proponer una reflexión sobre cómo ciertos elementos comunes al universo de la propaganda sirven para pensar en los flujos de producción de las fake news. Se considera aquí que el alineamiento de las fake news con las técnicas de la propaganda se produce en el procesamiento y almacenamiento de información, la elección del público objetivo y la consiguiente dirección de la distribución de la información adulterada, con el fin de modular el pensamiento de los seleccionados $y$, al final, afecta a la credibilidad periodística. Se parte de una conexión entre propaganda, algoritmos de inteligencia artificial y desorden informativo, especialmente cuando afecta a un público que, sin educación mediática, no dispone de técnicas básicas de verificación para llegar a la verdad de los hechos. Para la discusión en torno a este conjunto de fenómenos, que forma el corpus observado, se realizó una investigación bibliográfica para tensar las perspectivas teóricas que profundizan en esos temas. En los tiempos actuales, la propaganda especialmente en el campo político (con una gran ayuda del marketing digital), se ha sofisticado hasta el punto de asumir para sí la responsabilidad de proporcionar la base para la difusión de fake

\section{Abstract}

The issue is the emergence of fake news disturbing the democratic culture and discrediting journalism. The objective is to propose a reflection on how certain elements, common to the universe of propaganda, serve to think about the production flows of the fake news. It is considered, here, that the alignment of the fake news to the techniques of propaganda takes place in the processing and storage of information, the choice of the target audience and the consequent direction of the distribution of adulterated information, with the intention of modulating the thoughts of those selected and, in the end, affects journalistic credibility. For this purpose, a connection among propaganda, artificial intelligence algorithms and informational disorder is made. especially when it affects an audience that, without media education, does not have basic verification techniques to get to the truth of the facts. For the discussion around this set of phenomena, a bibliographical research was carried out, in order to tension theoretical perspectives that go deeper into these topics. Nowadays, propaganda especially in the political field (with strong help from digital marketing), has become sophisticated to the point of assuming responsibility for providing the basis for the spread of fake news and disinformation campaigns. 
news y campañas de desinformación.

Palabras clave: fake news, propaganda, Keywords: fake news, propaganda, journalistic credibilidad periodística, algoritmos, credibility, algorithms, artificial intelligence. inteligencia artificial.

\section{INTRODUCCIÓN}

Las llamadas fake news (FN) llegan a millones de personas cada día, perturbando la cultura democrática y desacreditando el periodismo. El adjetivo fake (falso) ni siquiera coincide con el sustantivo "news" (que, en este caso, significa "noticias"). Así, por razones obvias: para que un hecho se convierta en noticia, la prioridad, entre las diversas reglas éticas de la prensa, es que sea verdadero, es decir, una verdad factual.

Hannah Arendt señala que la verdad factual es pequeña, frágil, efímera. Como un primer registro de los acontecimientos, un primer -y precario- esfuerzo por saber lo que ocurre en el mundo, la verdad factual es más vulnerable a las falsificaciones y manipulaciones. Aun así, la verdad factual es fácilmente reconocible por todos, hombres y mujeres normales, comunes. [...] En el plano de los hechos, de los acontecimientos, de los eventos que todos vemos y que todos somos capaces de verificar y comprobar en el uso de las habilidades y facultades comunes a los seres humanos comunes, no hay nadie que no sepa divisar las distinciones entre la verdad factual y la invención deliberada de falsedades con el fin de ocultar los hechos. (Bucci, 2019, p. 22)

Las noticias falsas, por lo tanto, no son noticias, pero esto no impide que circulen o tengan consecuencias.

Además, siempre hemos tenido, en las redes sociales, rumores, perfiles fake o incluso usuarios de Internet con publicaciones ad hoc para engañar. Sin comprobar y a menudo sin leer, o sólo leyendo el título. O haciendo lo que es todavía más imprudente: a menudo, difundiendo historias inventadas a propósito, de interés para ciertas personas para atraer buzz o para atacar a otros. Todo eso resulta en un gran riesgo.

Wardle (2017, nuestra traducción) discurre sobre cómo se difunde este contenido fraudulento. Según ella, las personas comparten FN porque no verifican su contenido: "Parte de eso se está promoviendo por grupos que están deliberadamente intentando influir en la opinión pública, y otra se está difundiendo como parte de sofisticadas campañas de desinformación a través de redes de bots y fábricas de trolls". Las FN - tanto accionadas por humanos cuanto por bots (robots), programados por humanos, por supuesto - se propagan exactamente por donde está la mayoría excesiva del público objetivo (lo que deberá ser alcanzado): en las redes sociales y en los mensajeros instantáneos.

Como es fácil de presuponer, el propósito de la difusión de todo tipo de desinformación desde información truncada, impostora, hasta denuncias difamatorias - no es informar a la sociedad. Por el contrario, es exactamente falsificar, engañar, llevar a la ilusión. En otras palabras, busca llegar, de manera rápida y viralizada, a un determinado público, elegido por el análisis de los datos. Así, ese público objetivo difunde las FN entre sus pares (que piensan de manera similar) y, cuantas más personas se enteran, mayor es la reverberación del desorden 
de la información y aumenta la posibilidad de la intención de engañar y manipular con fines ideológicos.

Por lo tanto, como un timo, hacer que las personas repliquen la falsedad, a menudo conscientes de que no es verdad y sabiendo de antemano que están siendo engañadas. Cuando eso ocurre, es decir, cuando el hecho de compartir es deliberado, caracteriza una creencia arraigada en lo que es deseable que pase, algo reforzado por la intención de evitar la ansiedad. Algunos científicos, como Nickerson (1998), llaman a ese fenómeno un sesgo de confirmación, que connota la búsqueda o la interpretación de pruebas de manera parcial a las creencias existentes. En el fondo, hay una falta de formación en la vida para el autoconocimiento, para contar con mecanismos de enfrentamiento de conflictos íntimos, para dar lugar a momentos esenciales de escepticismo y para admitir considerar la idea del "lugar de habla" del pensamiento crítico interno.

Este artículo tiene como objetivo proponer una reflexión sobre cómo ciertos elementos comunes al universo de la propaganda sirven para pensar los flujos de producción de FN. Se considera aquí que el alineamiento de las FN con las técnicas de propaganda tiene lugar en el procesamiento y almacenamiento de la información, en la elección del público objetivo y en la consiguiente dirección de la distribución de la información adulterada, con el fin de modular el pensamiento de los seleccionados.

Los principios de la propaganda, un ámbito dedicado a la conquista de un determinado público - como, por ejemplo, cuando se hace un mapeo de los gustos de un grupo de personas, con el fin de conocerlos y poder así influir en ellos - resultan pertinentes para apuntar y manipular. Y eso es lo que hacen las FN. Así se recuperan elementos comunes a las estrategias de propaganda, especialmente aquellas utilizadas en los medios digitales - como el análisis, el monitoreo y el raspado de datos de perfiles, impulsando así la acción de los algoritmos de inteligencia artificial, provocando amenazas en el campo de la información - para debatir la cuestión de las FN y pensar cómo operan. La percepción de esa relación proviene de la concepción, usos y características propagandísticas con la similitud de las acciones desinformativas, especialmente en lo que se refiere al campo político. Tenemos conocimiento de que un algoritmo es una serie fija de etapas previamente determinadas, que un ordenador realiza para resolver un problema o completar una tarea, y está organizado para hacer cálculos exactos.

El procedimiento metodológico adoptado es la investigación bibliográfica, que ha permitido examinar lo que se ha discutido sobre ese conjunto de fenómenos.

\subsection{El periodista puede equivocarse, pero no produce fake news}

En principio, la mala fama de publicar FN recayó principalmente sobre los hombros de los periodistas, tal fue, y sigue siendo, la similitud estética entre las FN y las noticias. Es difícil de diferenciar, especialmente para los legos, quién de hecho persigue la ética periodística y se preocupa por la veracidad de la información, por los hechos de la vida cotidiana y, en consecuencia, por la credibilidad, de quién ni siquiera piensa en ello. En cualquier caso, se asume que un periodista no inventa noticias o que al menos no debería hacerlo. "Existe un acuerdo tácito entre quienes eligen esta profesión de periodista y el lector/oyente/telespectador que hace posible dar credibilidad al periodismo", afirma Traquina (2005, p. 19). Él añadió: el principal producto del periodismo contemporáneo, la noticia, no es 
ficción, es decir, los acontecimientos o los personajes de la noticia no son invención de los periodistas".

Pero incluso los medios de comunicación en los que se publican las FN se asemejan a los espacios tradicionales de la prensa, ya que se crean sitios web fraudulentos para hacerse pasar por periodísticos, con un diseño similar: además de la arquitectura de la interfaz, utilizan los mismos colores y fuentes, e incluso nombres similares a los de los medios existentes, a punto de confundir. En otras palabras, el tratamiento dado a las FN Ilevó a una asociación errónea de ese contenido fraudulento con el trabajo de los periodistas. Nos enfrentamos a (pseudo) textos de noticias, sin conexión con los hechos, que forjan la "imagen" de reportajes periodísticos. El público inocente es engañado, mientras que el público pícaro aprovecha la ola para consolidar sus puntos de vista, dentro de sus creencias y, así, no enfadarse y no tener desánimo psicológico.

"En efecto, la noticia debe ser entendida e interpretada como resultado de la consulta a fuentes plurales y diversas que ayudan a mirar el hecho desde distintos ángulos, a explicar su pasado y su contexto, y a descubrir sus consecuencias" (Restrepo y Botello, 2018, p. 9).

Ante una especie de inseguridad informativa - causada por la avalancha de FN - y diferentes expectativas en cuanto a la confianza en la prensa, parece necesario todavía reafirmar la ética periodística, reforzar las reglas de conducta tanto del editor como del reportero, y recordar la existencia de las entradas en los manuales de redacción y las discusiones interminables de las reglas de los linajes de investigación, para dejar claros los propósitos del periodismo.

En pleno siglo XXI, con una historia ya consolidada, podría parecer absurdo que el periodismo necesite probar su credibilidad y todavía fortalecer su deontología. Sin embargo, es notorio que, desde siempre, los medios de prensa se inclinan hacia determinadas corrientes ideológicas y, por lo tanto, reportan, interpretan y opinan como les conviene. Como señala Bucci (2000, p. 176), "impulsados por intereses turbios, hay propietarios de medios de comunicación y empleados de la cúpula de las empresas que patrocinan mentiras para lograr objetivos particulares". No hay exención, apartidismo o pluralidad, aunque se proclamen como principios editoriales de algunos medios, lo que en cierto modo alimenta la confusión entre periodismo y FN.

\subsection{Fake news y la plataformización}

Al considerar la economía política que mueve la Internet, es importante recordar que muchas de las tropas virtuales "se han convertido en un negocio". Bucci (2018a) recuerda que "los inventos calumniosos ahora producen poco dinero para algunos y millones para otros", dado que terminan deformando o incluso viciando los procesos decisorios en las democracias contemporáneas. Uno de los ejemplos más impactantes es la elección presidencial de EE. UU. en 2016, "una rocambolesca intriga de espionaje de hackers y conspiradores".

En ese momento, el término fake news se popularizó y se convirtió en politiquero gracias a Donald Trump, que comenzó a utilizarlo para rebatir, atacar e insultar a la prensa mainstream, con la que no estaba de acuerdo, después de ganar las elecciones en los Estados Unidos. Se considera que esas elecciones fueron manipuladas por acciones de marketing político o al servicio de intereses privados. A pesar del ardid de la campaña para persuadir a los británicos 
en las negociaciones del plebiscito del Brexit, para la retirada de Gran Bretaña del bloque europeo, la mayor resonancia de las FN fue provocada por Trump (Prado, 2019a, p. 166).

Para Bucci (2017), el hecho de que Trump acuse a las redacciones profesionales (como las de la CNN y del The New York Times) de difundir FN es, en sí mismo, un fraude. En la tribulación de la hiperinformación, dice él, "la prensa funciona como una especie de antídoto contra las FN, porque, por la forma como son identificadas y descritas, se demuestra que la prensa no es responsable de fabricarlas. Trump miente al establecer la isonomía entre las FN y la prensa".

Es necesario señalar que la difusión de las FN se produce especialmente en las plataformas de redes sociales. En una entrevista al periódico brasileño Folha de S. Paulo (Sá, 2020), Campos, profesor de la Universidad de Frankfurt y considerado "una de las principales voces en el debate sobre el proyecto de ley de las fake news", dijo: "Las elecciones estadounidenses en 2016 y otros eventos globales han dejado clara la posición central de las plataformas [digitales] como la nueva infraestructura de comunicación, con un nítido impacto en la democracia". Él añadió: "Las plataformas crean espacios públicos a partir de relaciones privadas y, en este sentido, son decisivas para la formación de la opinión pública dentro de las democracias".

El esquema de plataformización de los grandes conglomerados monopolísticos no es transparente. Veremos a continuación la acción algorítmica. "El error fue asumir que las plataformas estaban del lado solamente de la democracia. Cuando, de hecho, eran herramientas de transformación y disrupción, y no sólo los regímenes autoritarios podrían ser amenazados, sino también la democracia", dijo Martin Moore en una entrevista a la agencia de periodismo investigativo brasileña Agência Pública (Rudnitzki, 2019). Él añadió:

Puede parecer fantástico que estemos viendo cómo se está incluyendo a los outsiders, pero algunas personas e ideas pueden estar siendo excluidas aún más. El problema es que la estructura de ese mundo digital favorece a ciertas prácticas e individuos en detrimento de otros, particularmente aquellos que están dispuestos a romper las convenciones y son más propensos a atacar, a provocar choque y revuelta, a llamar la atención y a enfrascarse en formas de extremismo.

Él recordó una acción de Facebook considerada una de las más impactantes: la manipulación de los 87 millones de datos retenidos de los usuarios:

Me he basado en Robert Mercer [el empresario detrás del escándalo de Cambridge Analytica] para eso. Él no tenía ningún estatus o posición política. Aun así, gracias a su dinero y a su conocimiento en el área [de big data], fue capaz de invertir y comprender el uso político de los datos en masa para influir en las elecciones estadounidenses [en 2016]. Por supuesto, la gente con mucho dinero podía influir en las elecciones en el año pasado, pero no era tan efectivo e impactante como lo que se hizo en los últimos años con las herramientas digitales. (Moore, 2019)

Cuando se le preguntó por qué los agentes disruptivos fueron más favorecidos que otros en las plataformas, en las elecciones estadounidenses de 2016, Moore respondió a la reportera Ethel Rudnitzki (2019):

El problema está en la forma como se hace la publicidad en las plataformas, lo que fomenta cualquier cosa para generar clics. Las redes sociales son mejores para provocar respuestas de comportamiento en las personas, más que la reflexión y el 
convencimiento. Así que la gente que sigue las tradiciones democráticas e intenta persuadir a la gente a una causa, intentando convencerlos de que voten por un determinado candidato, no ha sido favorecida. El sistema favoreció a aquellos que buscaron respuestas inmediatas y emocionales y no involucrarse en el debate democrático.

Especialmente en los albores del siglo XX, la propaganda, especialmente en el ámbito político, perseguía el perfil potencial de los consumidores de medios impresos y electrónicos: votantes y formadores de opinión entre los lectores de periódicos; luego, oyentes (1920); y luego, telespectadores (1940). Pero fue a mediados de la década de 1990, con el advenimiento de la Internet - (inicialmente) abierta y flexible y, principalmente, hiperpoblada de manera veloz -, que el área encontró un terreno fértil para expandirse, no sólo con la persuasión latente atacando al público incauto, sino también manipulando de manera a influir en él para que actuara de acuerdo con su propio objetivo, a menudo de soslayo.

\section{INTERACTIVIDAD EN LA WEB, TERRENO FÉRTIL PARA IMPLANTAR TÉCNICAS PUBLICITARIAS}

Paralelamente a la fase de interactividad de la Internet (web 2.0), todo tipo de colaboradores y participantes ganó espacio y voz. Desde simples comentarios hasta opiniones en blogs, el contenido generado por el usuario se instala sin retorno. Una vez más, cabe destacar que ni siempre con preocupaciones éticas o patrones y reglas editoriales, porque éstas son propias de los profesionales del periodismo.

Así uno de los cambios cruciales del periodismo en Internet son sus modos de circulación, es decir, la velocidad con que circula la información. Lo que no cambia en el periodismo, ya sea en la red o fuera de ella, es el esquema de la producción de noticias: la planificación del reportaje, la investigación del tema, la búsqueda de fuentes y de personajes, la investigación, la verificación, la identificación, la recopilación de información, la redacción, la revisión (ya sea hecha por el propio reportero o editor) y la edición.

Al ser permeada de técnicas de propaganda, destinadas a conocer y trazar el perfil de personas, la red mundial de ordenadores no sólo fue destinataria sino también se tornó un prolífico campo de monitoreo, análisis y especificación de datos. Según Perón (2018), la captura de datos es "una entrada que puede darse de diversas formas (datos de geolocalización, de comportamiento), por los más diversos sensores (cámaras, móviles, sensores biométricos) y a partir de la programación que se les imputa, lo que les permite correlacionar y producir nueva información, como características de consumo, patrones de relación, etc.".

De extremo desvelo con que se da ese monitoreo y el consiguiente análisis de datos es la transparencia en la propaganda y la financiación política, como señala Moore (Rudnitzki, 2019): “Es necesario saber quiénes son los destinatarios de los anuncios y también qué datos de los usuarios se han agrupado y utilizado, además de la necesidad de regulaciones a ser implementadas, como la prohibición de dirección de anuncios basados en datos personales por los anunciantes".

Sin embargo, en el fenómeno de la información de datos, con la falta de tratamiento legal, la lógica económica que rige las plataformas permanece intacta. 
Santaella tiene razón al recordar que "las estrategias de seducción y persuasión de la publicidad siempre han funcionado" para atrapar el público. "Son mensajes de fuerte atractivo visual, cuyos titulares son tan increíbles que se vuelven irresistibles. En las redes, esos mismos principios siguen presentes" (2018, p. 30).

Borel, a su vez, destaca que a las noticias también se atribuye el propósito de entretener. Sin embargo, en su opinión, esa intención debería dejarse de lado porque abre el camino a las distorsiones: "La publicidad basada en clics nos ha dejado a la deriva en un mar de titulares inexactos y sensacionalistas, incluso en las agencias de noticias legítimas; eso facilita la supervivencia de titulares de noticias dramáticos y falsos" (2017, nuestra traducción).

\subsection{Opiniones de todos los lados}

Reunimos a no profesionales, en reparto sociotécnico, produciendo información -sin investigación, verificación, fuentes, etc., distribuida en la blogosfera, en sitios web y en cajas de comentarios, y luego en redes sociales y mensajes instantáneos - dirigida a aquellos que están abiertos a recibir cualquier material, principalmente de sus pares, que son, en principio, considerados confiables. Así, experimentamos la conflagración de opiniones tan disparatadas como factibles - notas fecundas e ingeniosas a merced de los expertos en propaganda, especialmente la maliciosa.

En la era de opiniones y convicciones proliferadas por todos y para todos, vale la pena recuperar las consideraciones de Peirce - esbozadas en el siglo XIX, pero aún muy actuales que nos ofrecen la siguiente reflexión:

Si el establecimiento de la opinión es el único objeto de la inquisición, y si la creencia es de la naturaleza de un hábito, ¿por qué no alcanzaríamos el fin deseado tomando cualquier respuesta a una pregunta de nuestra simpatía, y reiterándola constantemente a nosotros mismos, aferrándonos a todo lo que pueda llevar a esa creencia, y aprendiendo a mirar con desprecio y odio lo que pueda perturbarla? (1877)

Las deducciones peircianas nos ayudan a pensar en la era de la tecnología de la información (especialmente aquella relacionada al sentido de la información como relación con los datos) y su reciente crisis: la importancia de la integridad de las creencias es mayor que cualquier otra creencia particular, y es tan inmoral como desventajoso evitar mirar el fundamento de cualquier creencia, por temor a que se revele podrido. "La persona que confiesa que existe algo como la verdad, que se distingue de la falsedad simplemente por esto, que, si continuada, nos llevará al punto que deseamos alcanzar y no por un mal camino, y luego, aunque convencida de esto, no se atreve a conocer la verdad, sino que busca evitarla, se encuentra en un lamentable estado de ánimo (Peirce, 1877)".

En las redes y los mensajeros instantáneos, sólo se acerca uno que esté de acuerdo con la posición del otro. De lo contrario, la prohibición es inmediata y, en muchos casos, corriente, tras el uso de lenguaje ofensivo. La enormidad del espacio de la red virtual, como en todo, sólo refuerza las acciones, las conexiones, con informaciones confirmatorias. Así, el cambio de actitud de las personas atingidas por la desinformación, creando desconfianza, y la atmósfera confusa causada por las FN en los medios sociales influyen en la participación en la política y la cultura. 
Gala y Baldi (2019, p. 243) afirman aún: "Los ámbitos de las noticias, de la información y de la ciencia se ven así desafiados a experimentar con nuevas formas de presentar, analizar, señalar y difundir lo que parece amenazar su credibilidad". "La lógica del pensamiento crítico", ellos siguen adelante "como también se destaca en el trabajo epistemológico de Peirce, es la piedra angular para combatir y prevenir la distorsión de los hechos y de los conocimientos establecidos. Se trata ahora de tener en cuenta cómo ese sentido crítico debe también instalarse cada vez más en el ámago de los softwares que generan y producen la información en red".

Es bueno subrayar que la propuesta no es hacer la reflexión sobre apenas uno de los temas levantados hasta aquí: propaganda, ética y credibilidad periodística, minería y manipulación de datos o incluso algoritmos, IA, FN y sus creencias, sino establecer una conexión entre esos elementos para intentar comprender la esfera algorítmica actual.

\section{CULTURA ALGORÍTMICA DE ALTERACIÓN INCESANTE}

"Los algoritmos se basan en las propias elecciones que hacemos, diseñan las predilecciones que reportamos en las redes. Por lo tanto, ya no se trata apenas de demonizar el poder de las redes", dice Santaella, "porque ellas no hacen más que devolvernos el retrato de nuestras mentes, deseos y creencias" (2018, p. 16).

Las plataformas de redes sociales, donde la comunicación de masa llegó, utilizan algoritmos para filtrar y priorizar el contenido de cada usuario en base a varios indicadores, como el comportamiento de exhibición y las preferencias de contenido. La desinformación diseñada para provocar una reacción emocional puede florecer en estos espacios cuando los algoritmos detectan que es más probable que un usuario se involucre o reaccione ante un contenido similar (Wardle, 2017). Muchos de los datos imputados son parciales y rankeados al ser clasificados y ramificados por algoritmos.

La inteligencia matemática propia de los algoritmos puede optimizar las búsquedas en Internet y hacer recomendaciones personalizadas según nuestro perfil, definir y mostrar anuncios en las páginas que visitamos, etc. Además de dirigir los anuncios, algunas plataformas también dirigen el contenido que se mostrará de forma prioritaria. Estos pueden variar desde resultados de búsqueda hasta noticias compartidas por contactos en redes sociales (CGI.BR, 2019). En esto, cuando el algoritmo incluye y excluye datos, lo que es opuesto e incómodo es apartado, en una acción típica de intento de modulación de comportamiento de los usuarios.

"El procesamiento de datos en un ordenador se limita exclusivamente a manipulaciones estructurales de datos y se hace por medio de programas. Estos son siempre funciones matemáticas y, por lo tanto, también son 'datos'. Ejemplos de estas manipulaciones en el caso de los textos son el formato, el orden, la comparación con otros textos, estadísticas de palabras utilizadas y su entorno, etc." (Setzer, 2015).

En una de las observaciones, es posible detectar que, como muchas personas están conectadas entre sí - y necesitan estarlo porque la proliferación de información es muy grande- y como todo cambia todo el tiempo, ellas necesitan ayuda para separar lo que es verdad de lo que no lo es. Incluso porque la circulación desenfrenada de información pierde valor en las redes. Son personas (o voces) que, por primera vez, han tenido la oportunidad de exponerse, hablar, dar su opinión. Sin embargo, no todas tienen discernimiento sobre 
educación para los medios de comunicación. Así, se forman burbujas o grupos autorreferenciales, en el mismo sentido de las burbujas que ya existen en la vida fuera de las redes, sólo que más radicales y en mayor proporción. Al final es necesario filtrar dentro de la abundancia y la velocidad de la información. En general, no se dan cuenta de lo ideológicamente segregadas que están.

Pero, en la era de las redes sociales, en la condición tanto de ventaja como de desventaja de la entrada de la ingeniería de los opacos algoritmos, el individuo es, para Bucci (2018, p. 28), espejado y reflejado todo el tiempo por las multitudes que lo aprisionan, lo encapsulan. "Son las multitudes de iguales, las multitudes especulares, las multitudes de mismos". Sin embargo, las orientaciones de los algoritmos de las redes sociales, en continuo perfeccionamiento, son la estimulación y la fortificación de las burbujas. Los algoritmos hacen más espesas lo que Bucci llama "murallas", que tienen como objetivo separar las burbujas. Para él, existe el hecho agravante de que "esos algoritmos son cerrados en códigos propietarios, de tal manera que los sistemas que regulan el flujo de información en la práctica no son públicos". Así pues, la red tecnológica a través de la cual transita la información, que debería ser neutra, no lo es". Para los que se ocupan de los algoritmos, no importa cuál sea el resultado. Obviamente, sólo los humanos que establecen los inputs tienen la capacidad cognitiva de escrutar.

Al considerar la perversa economía de los datos, encubriendo o no la entropía que involucra los datos, Bentes $(2019$, p. 222) explica la cuestión del compromiso: "Para operar la prosperidad financiera en esta lógica de acumulación, es esencial que los servicios digitales capten y movilicen la atención de los usuarios para que pasen el mayor tiempo posible conectados en sus plataformas. Ella añade:

Pues, cuanto más tiempo pasen enganchados y enfrascados, mayor será la producción, recopilación y almacenamiento de datos, y, por lo tanto, mayor será la agudeza de predicción de los mecanismos algorítmicos, lo que a su vez aumentará el valor de los ingresos del servicio. En ese sentido, en la economía digital, el valor de los datos está intrínsecamente conectado al valor de la atención. Por consiguiente, las estrategias de este mercado se centran en el desarrollo de mecanismos persuasivos para captar la atención, en los que la gestión algorítmica desempeña un papel central.

La lógica algorítmica en la plataformización de la economía prevé ciclos de anticipación. "Así pues, la capacidad de predecir, influir y guiar el comportamiento humano mediante la gestión de algoritmos es también, en cierta medida, la capacidad de captar, movilizar y dirigir la atención de los usuarios" (Bentes, 2019, pp. 222-223).

\subsection{Carácter rastreador del algoritmo}

En la medida del desorden, para Cardoso (2019), la cuestión de la invisibilidad de los mediadores se despliega en la aparente neutralidad de los algoritmos, que, dado el fuerte carácter indicativo de sus signos - que dirigen a los usuarios a los resultados relevantes, apuntando al contenido referido y no al proceso de selección-, no se muestran como medios en las prácticas que agencian y promueven implícitamente una percepción general en la cultura digital de centrarse en los resultados, y no en los procesos que elaboran una mediación de los resultados. "Una de las características más destacadas de la cultura del algoritmo", él añade: es su carácter rastreador, es decir, su archivamiento de registro de acciones. Así como un detective pretende rastrear las acciones para luego conectarlas para elaborar 
conocimiento, los sistemas algorítmicos también pretenden recopilar todo tipo de datos para poder elaborar su procesamiento, su conocimiento y su capital (id.)

Asociadas con la digitalización, la captura y la consiguiente minería de datos son el proceso desarrollado cuando se desea monitorear grandes volúmenes de datos combinando herramientas de estadística e IA para reconocer patrones útiles. Es necesario comprender las tecnologías disruptivas, como la propia IA.

\section{DISCUSIONES Y RESULTADOS CONJETURADOS}

Los algoritmos inciden en opacidad y, al decidir por los humanos, los códigos algorítmicos son turbios. Sin embargo, los científicos que se ocupan de IA tienen la convicción en la posibilidad de que las máquinas simulen el cerebro humano, lo que implica la intención de los procesos de aprendizaje. Gillespie afirma que,

a medida que adoptamos herramientas computacionales como nuestros principales medios de expresión y comenzamos a hacer uso no sólo de las matemáticas, sino de toda la información digital, comenzamos a someter el discurso y el conocimiento humano a esas lógicas de procedimiento que soportan toda la computación. $Y$ hay implicaciones específicas cuando usamos algoritmos para seleccionar lo más relevante a partir de un corpus de datos compuesto por rastros de nuestras actividades, preferencias y expresiones. [...] Casi todas las actividades públicas incluyen el almacenamiento de extensos registros, catalogación y archivamiento de documentos - y lo hacemos aún más en las redes de comunicación proyectadas, de modo que cada entrada, cada página visualizada y cada clic dejen un rastro digital. (2018, pp. 97-99)

La IA describe programas informáticos "entrenados" para resolver problemas que normalmente serían difíciles de resolver para un ordenador.

Esos programas "aprenden" de los datos analizados, adaptando métodos y respuestas para maximizar la precisión. A medida que la desinformación crece en alcance y sofisticación, algunos buscan la IA como una forma de detectar y moderar el contenido de manera efectiva. La IA también contribuye al problema al automatizar procesos que permiten la creación de manipulaciones más persuasivas de imágenes visuales y posibilitan campañas de desinformación que pueden ser dirigidas y personalizadas de manera mucho más eficiente. (Gosh como se cita en Wardle, 2018)

"Las empresas pasaron de la gestión de productos a la gestión de clientes, compilando bancos de datos sobre clientes individuales para que puedan conocerlos mejor y desarrollar ofertas y mensajes personalizados", refuerzan la acción personalizada Kotler y Keller (2005, p. XVIII).

Diferentes formas de ejecutar algoritmos exponen ciertos asuntos mientras que ocultan otros. Los científicos de la computación producen algoritmos como objetos conceptuales indiferentes a los detalles de implementación, mientras que los llamamientos a la responsabilización promueven algoritmos como cajas cerradas a ser abiertas, tal y como lo entiende Seaver (2017, p. 1, nuestra traducción). El autor sugiere "pensar en algoritmos no 'en' cultura, sino 'como' cultura: partiendo de patrones generales de significado y práctica que puedan ser empeñados y empíricamente involucrados". 
Es visible que, a partir de la constatación de la contaminación de la comunicación causada por el exceso de información producida y reproducida por muchos que no tienen una educación mediática crítica, y no sólo por profesionales, el desorden informativo infecta toda la esfera de la comunicación, que se ve dañada en su confianza y reputación. Eso también se debe a la ascensión de la guerra políticamente polarizada - incluso con recurso de disparos masivos de mensajes - por las FN, resultando a menudo en la incitación al discurso de odio y a la promoción del perjuicio, en un área sensible del ciberespacio (en el sentido bélico del término, cuando él surgió).

Cuando sucede la emergencia del disturbio de la información, sobre todo con la planificación profesional en relación con el contenido difamatorio - momento adecuado para producirlo y exactamente a quién dirigirse - la base de comunidades y burbujas ya estaba preparada a escala. Bastaba con aprovechar el proceso en el que se imputan las premisas en los algoritmos de IA, esbozado para uso de la información como estrategia de poder, escondido detrás de lo que se llama "la mejora de nuestra experiencia", es decir, la experiencia del público.

En la sobrecarga de información, particularmente cuando nuestros cerebros están agotados, "los mensajes coordinados y consistentes nos engañan fácilmente", dice Wardle (2017, nuestra traducción), ya que "nuestros cerebros dependen cada vez más de la heurística debido a la enorme cantidad de información que parpadea ante nuestros ojos cada día". Ella concluye: "Cuando vemos varios mensajes sobre el mismo tema, nuestro cerebro lo utiliza como un atajo para la credibilidad. Deberíamos decir que sí - ya he visto la misma afirmación varias veces hoy". Wardle aún conjetura que, cuando la gente siente miedo y rabia, disminuye su capacidad de pensar críticamente.

Sin embargo, comunicarse es una condición fundamental de la vida. En el torbellino de noticias difundidas por cualquier persona, profesional o no - en este último caso, sin temor a las normas de conducta (a menudo intencional) - la información escapa a la verdad factual, como las propagadas disfrazadas, los errores, las falsedades, la mala fe, etc. La información circula e intermedia en los sistemas computacionales: en redes sociales, mensajería instantánea y sitios web dudosos, junto con la prensa mancomunada con facciones - en el sentido de aquellos que planean la destrucción de adversarios. En ese sentido, provoca el desorden informativo que nos rodea y, sobre todo, rodea a las personas con tendencia a sufrir influencias de las FN, porque no entienden los procesos tecnológicos y las posibles formas de control de lo que reciben debido a la total falta de educación para el consumo de los medios.

Santaella $(2018$, p. 27$)$ subraya que no basta con tener en cuenta a los intermediarios de la información, considerando los medios tradicionales o las redes sociales, "como los únicos responsables de los problemas". Por el contrario, "es una tarea colectiva, nada fácil, pide acciones creativas como antídotos a la propaganda engañosa, a los discursos de odio, a los contenidos prejuiciosos y a las noticias falsas". La autora destaca el "tipo más perjudicial de este elenco falsificador", que se puede encontrar "en las propagandas intencionadamente engañosas con el fin de promover puntos de vista tendenciosos, casi siempre para alimentar causas y programas políticos". Y añade: "Es precisamente ese campo de acción y decisión del que dependen los destinos de la democracia en esta era posdigital" (Santaella, 2018, p. 35).

Al aprender - profundamente o no - con la máquina, bots, chatbots y cíborgs aumentan esos desarrollos invasivos, ayudando a falsear, replicar y viralizar en las redes y mensajerías 
instantáneas, contenidos de interés específico, producidos con meticulosa precisión de acuerdo con el resultado del análisis de los datos, para dirigir de manera algorítmica a los usuarios de Internet, siempre y cuando sean influenciables e indecisos (Prado, 2019, p. 70).

De hecho, ¿cómo los desarrolladores establecen el algoritmo para la toma de decisiones, aunque virtualmente automatizados? Definiciones de parámetros, reconocimiento de patrones, cálculos, alineación de valores, inteligencia aumentada... El hecho de que los datos públicos no estén en la lógica de almacenamiento de datos abiertos pone trabas al menos a uno de los lados de las posibles soluciones. Las preguntas permanecen.

Gala y Baldi conjeturan sobre la actualidad:

Nos encontramos, por lo tanto, ante una peculiar superposición de conflictos desencadenados por un discurso público radicalizado y fragmentado, donde los límites entre propaganda, alteración de la realidad, conocimiento establecido e invención de hechos parecen evaporarse, desafiando una construcción de la realidad basada en noticias y comentarios virales, constantemente percibidos como sospechosos y acusados de ser manipulados. (2019, p. 244)

Aumentar la visibilidad de la mala información es una tarea minuciosa que implica, en primer lugar, ofrecer una educación mediática para impulsar la población a saber utilizar las tecnologías de la información y a obtener habilidades de reconocimiento factual y a saber interpretar y analizar los mensajes para adquirir la capacidad de distinguir lo que es falso (o imitativo del estilo periodístico), en la intersección de la tecnología con la enseñanza, fomentando una educación mediática desde la infancia para que crezcan sabiendo desconfiar y, en consecuencia, verificar FN; pasando por los adolescentes, principalmente, por ser los más enganchados a la vida en las redes, por la inmediatez desenfrenada, rodeándose de actos contestatarios (propios de ese grupo de edad) ante la gran falta de respeto al derecho a la información que todos deben tener; hasta, y sobre todo, la población de mayor edad, que son (por los resultados de la investigación) los que más replican FN; promoviendo así la implicación crítica con respecto a lo que fácilmente se les ofrece en el entorno numérico, con la intención de que se lo piensen dos veces para una mejor evaluación crítica.

Entre las opciones de la propuesta de Aguaded y Romero-Rodríguez para una necesaria reformulación de los medios desde las audiencias, están: "Educación mediática, digital e informacional desde una política pública activa que fomente la formación crítica, participativa y creativa de la ciudadanía. Promoción de programas educativos que conjuguen la formación crítica con el periodismo interpretativo y de investigación" (2015, p. 53).

Mientras la sociedad - y especialmente la comunidad periodística, que recibe críticas de todos lados, como si estuviera actuando en el epicentro del problema de la desinformación - esté a merced del empeoramiento de los sistemas de vigilancia y moderación de la información basados en algoritmos de IA para interferir y modular el pensamiento de un público designado (preferentemente vulnerable), estaremos atados de manos en el sentido de tener un freno ante esa intrusión, que viene de los sitios web especializados en FN (a veces sensacionalistas), pero especialmente de las redes y sus plataformas más rentables - Facebook (con él, WhatsApp e Instagram) y Google (incluyendo YouTube) - con millones de visitas por segundo, en las que se encuentra gran parte de la gente y que pasan por una era de distribución (por un lado, con control de visualizaciones a su antojo $y$, por otro, totalmente desordenada de 
información, porque cualquier absurdo procedente de las brigadas web se produce para ser publicado).

"Con su capacidad para reunir datos que permiten actualizaciones en un programa de marketing en la Web, Google sostiene un estilo de marketing en el que los recursos y el presupuesto de propaganda pueden ser constantemente monitoreados y optimizados", dicen Kotler y Keller (2005, p. 29).

Los actuales esquemas de generadores de FN, en pleno siglo XXI, han bebido en la fuente de funcionamiento de la propaganda tradicional, pero obviamente, con los recursos tecnológicos, han elevado todas las fases del proceso de modulación de público seleccionado. Son ellas:

- recopilación y almacenamiento de información - más ágiles y utilizando grandes cantidades de datos;

- análisis destinados a refinar, elegir el público a manipular (aquí, en este recorte, por la FN) - con un acceso cada vez más fácil proporcionado por los propios perfiles que caen en una variedad de trampas: desde el acuerdo con los términos de condiciones de uso, pasando por la aceptación de cookies de rastreo, hasta la respuesta a tests de personalidad y comportamiento que inundan las redes $-\mathrm{y}$

- dirección, difusión y circulación - que, con la ayuda de robots, causan disparos masivos, y replican y viralizan los perfiles falsos.

Todas esas etapas que desequilibran la información precisa, a favor de intereses - en general, turbios, porque estamos hablando de FN - se aceleran y en una escala mucho mayor de lo que veíamos en el pasado, con las técnicas de propaganda, antes de la posibilidad de utilización del ciberespacio para ese tipo de acciones perturbadoras, que crean un desorden informativo, también en mayor proporción, cuando el alcance mismo de la propaganda era menor, pero aun así, llegando a un determinado público potencial.

\section{CONCLUSIONES}

En los tiempos actuales, la propaganda, especialmente en el campo político (con ayuda del marketing digital), se ha sofisticado hasta el punto de asumir para sí la responsabilidad de proporcionar la base para la difusión de FN.

Una plataforma descentralizada con el objetivo de utilizar la tecnología blockchain, resistente a la modificación de los datos que contiene, lo que también es útil cuando es necesario proteger el contenido noticioso de la adulteración y la censura, en nombre del periodismo, es encomiable. Además, hacer que la información de una red independiente de redacciones sea más fiable es algo que prevalece en tiempos de reputación en baja debido a la desinformación desenfrenada. Es imprescindible recordar que cualquier integrante o usuario de la cadena puede tener acceso al banco de datos, pero se justifica que soluciones auditables, producidas, intercambiadas y analizadas continuamente también deben entrar en un modo de redoblada vigilancia constante (Prado, 2020).

Sólo para que conste: en la época en que las gacetas comenzaron a influir en los lectores, aunque haya sido otro momento y no el periodismo que se configuró entre finales del siglo XIX y principios del $X X$, podríamos explicar más a menudo sobre ética periodística, 0 específicamente a partir del siglo XVII, cuando los políticos utilizaban sistemáticamente la prensa para dirigir la sociedad con periódicos creados - o en conspiración - para exaltar a los 
partidos políticos y/o determinados candidatos. Era necesario demostrar cómo funcionaba el periodismo, porque uno no tenía idea del propósito. Sin embargo, en pleno siglo XXI, podría parecer absurda la necesidad de que el antiguo periodismo, el tradicional, impusiera respeto, demostrara credibilidad y siguiera resonando que la deontología periodística es intrínseca a la profesión. Sin embargo, no es tan absurdo, es notorio que desde siempre (y hasta hoy) algunos medios de comunicación tienen inclinación para ciertas corrientes ideológicas y, por lo tanto, reportan, interpretan y opinan como les conviene.

\section{Referencias}

Aguaded, I, Romero-Rodríguez, L.M. (2015). Mediamorfosis y desinformación en la infoesfera: Alfabetización mediática, digital e informacional ante los cambios de hábitos de consumo informativo. Education the Knowledge Society, vol. 16, núm. 1, abril, 44-57. http://dx.doi.org/10.14201/eks20151614457.

Bentes, A. (2019). A gestão algorítmica da atenção: enganchar, conhecer e persuadir. In: Polido, F., Anjos, L., Brandão, L. (Ed/s.). Políticas, internet e sociedade. Iris (Instituto de Referência em Internet e Sociedade), 222-234. http://bit.ly/3rgDeWt.

Borel, B. (2017, 4 de enero). Fact-checking won't save us from fake news. FiveThirtyEigth. http://53eig.ht/3rhupvu.

Bucci, E. (2000). Sobre ética e imprensa. Companhia das Letras.

Bucci, E. (2017, 6 de julio). A luta livre contra a imprensa. O Estado de S. Paulo. http://bit.ly/3mCQq4D.

Bucci, E. (2018). Pós-política e corrosão da verdade. Revista USP, n. 116, pp. 19-30, ene./mar. 10.11606/issn.2316-9036.v0i116p19-30.

Bucci, E. (2018a, 24 de mayo). Não sabem o que é 'news' e querem caçar 'fake news'. O Estado de $S$. Paulo. http://bit.ly/3h3Wh1w.

Bucci, E. (2019). Existe democracia sem verdade factual? Estação das Letras e Cores.

Cardoso, T. (2019, 23 de marzo). Humanidades digitais e agenciamento algorítmico. Transobjeto. http://bit.ly/3mGnrN8.

CGI.BR. (2019). Internet, democracia e eleições: Guia prático para gestores públicos e usuários. http://bit.ly/2KdS67v.

Gala, A.C.O.S., Baldi, V. (2019). Quem averigua as notícias, os algoritmos ou jornalistas. A lógica crítica de C. S. Peirce como processo de identificação de uma fake news. Ámbitos. Revista Internacional de Comunicación, n. 46, 241-260. 10.12795/Ambitos.2019.i46.13.

Gillespie, T. (2018). A relevância dos algoritmos. Parágrafo, v. 6, n. 1, 95-121, ene./abr. http://bit.ly/2KqES7l.

Kotler, P., Keller, K. L. (2005). Administração de Marketing. 14a ed. Pearson Education do Brasil, 2012.

Nickerson, R.S. (1998, Junio). Confirmation bias: a ubiquitous phenomenon in many guises. Sage Journals Pub, v. 2, n. 2, 175-220. 10.1037/1089-2680.2.2.175.

Peirce, C.S. (1877). A fixação da crença. Biblioteca On-line de Ciências da Comunicação. https://bit.ly/38qSg3c.

Prado, M. (2019). Inteligência artificial na cultura informativa e algoritmos de enganação. SANTAELLA, Lucia (Org.). Inteligência artificial \& redes sociais (pp. 57-72). EDUC- Editora da PUC-SP.

Prado, M. (2019a) Redes sociais digitais e a esfera pública: "fake news" e a manipulação da opinião coletiva. MARTínEZ-ÁVILA, Daniel; SOUZA, Edna Alves de; GONZALEZ, Maria Eunice Quilici (Orgs.). Informação, conhecimento, ação autônoma e big data: continuidade ou revolução? (pp. 165-184). Oficina Universitária, Cultura Acadêmica.

Prado, M. (2020). Blockchain como antídoto às fake news. SANTAELLA, L. (Org.). A expansão social do 
blockchain. (pp. 77-96). Educ - Editora da PUC-SP.

Restrepo, J.D., Botello, L.M. (2018). Ética Periodística en la Era Digital. International Center for Journalists. https://bit.ly/37HljAo.

Rudnitzki, E. (2019, 22 de octubre). Martin Moore: Se não fizermos nada, vamos caminhar para a democracia das plataformas. Agência Pública. https://bit.ly/3h6GTBv.

Sá, N. (2020, 23 de septiembre). Afastar a aplicação do direito na internet é entusiasmo anacrônico, diz especialista. Folha de S. Paulo. http://bit.ly/3pdxAmm.

Santaella, L. (2018). A pós-verdade é verdadeira ou falsa? Estação das Letras e Cores.

Seaver, N. (2017). Algorithms as culture: some tactics for the ethnography of algorithmic systems. Big Data \& Society, v. 4, n. 2, jul./dec. pp. 1-12. 10.1177/2053951717738104.

Setzer, V.W. (2015, 25 de mayo). Dado, informação, conhecimento e competência. Ime.usp.br. https://bit.ly/2LK62Gv.

Traquina, N. (2005). Teorias do jornalismo: Porque as notícias são como são. Insular.

Wardle, C. (2017, 16 de febrero). Fake news. It's complicated. First Draft. http://bit.ly/3palFnV.

Wardle, C. (2018). Information disorder: The essential glossary. Harvard Kennedy School. Shorenstein Center on Media, Politics and Public Policy. https://bit.ly/3rijh1s.

\section{Semblanza de la autora}

Periodista y profesora de Posgrado de la Facultad de Sociología Política de São Paulo. Estudiante postdoctoral en el Departamento de Información y Cultura, Escuela de Comunicaciones y Artes, Universidad de São Paulo (USP). Doctora en Comunicación y Semiótica y Máster en Tecnologías de Inteligencia y Diseño Digital, por la Pontificia Universidad Católica (PUC-SP), donde creó e enseñó en el curso de extensión Tecnologías Digitales y Autoría en la Red. Postgraduada en Comunicación Periodística y licenciada en Periodismo por la Faculdade Cásper Líbero (SP), donde fue profesora de los cursos de Radio, TV e Internet y Periodismo, de 2003 a 2018. Fue profesora de la Escola Superior de Propaganda e Marketing (ESPM-SP), de 2011 a 2017, en el curso de Periodismo y en el Máster Profesional en Producción y Mercado Periodístico. 